\title{
Scattering functions of polyhedra
}

\author{
Byeongdu Lee
}

9700 S. Cass Ave., Lemont, IL 60439, USA, blee@anl.gov

Due to recent advances in materials synthesis, various polyhedral nanoparticles are available for researches and applications. Not only particles, but also pores in a crystalline materials can be polyhedron shaped. In the scattering point of view, scattering calculation of the particles is not much different from that of pores, it is just a matter of contrast inversion. Thus, the functions for particles can be directly used for the interpretation of pores in small angle scattering. Nevertheless, as the resolution of measurement increases, there has been more demand for the fast and accurate computation of scattering from polyhedral particles and pores.

In this presentation, derivation of the scattering functions of regular and semi-regular polyhedra will be discussed. The method is based on the classical volume integration, but can be further extended to use Stokes' theorem. Solutions for the singularities of the functions will be presented too. Lastly, as an example, we will present assembly of polyhedral nanoparticles with DNA ${ }^{1-2}$ and determination of relative orientations of the particles in an assembly using SAXS.

\section{Reference}

1. Laramy, C. R., et al., (2019). ACS Nano, 13, 1412.

2. O'Brien, M. N., et al., (2015). Nat. Mater., 14, 833. 